\title{
Decompressive craniectomy in subarachnoid hemorrhage
}

\author{
Erdem Güresir, M.D., Patrick Schuss, M.D., Hartmut Vatter, M.D., Ph.D., \\ Andreas RaAbe, M.D., Ph.D., Volker Seifert, M.D., Ph.D., and Jürgen Beck, M.D., Ph.D.
}

Department of Neurosurgery, Johann Wolfgang Goethe-University, Frankfurt am Main, Germany

\begin{abstract}
Object. The aim of this study was to analyze decompressive craniectomy (DC) in the setting of subarachnoid hemorrhage (SAH) with bleeding, infarction, or brain swelling as the underlying pathology in a large cohort of consecutive patients.

Methods. Decompressive craniectomy was performed in 79 of 939 patients with SAH. Patients were stratified according to the indication for DC: 1) primary brain swelling without or 2) with additional intracerebral hematoma, 3) secondary brain swelling without rebleeding or infarcts, and 4) secondary brain swelling with infarcts or 5) with rebleeding. Outcome was assessed according to the modified Rankin Scale (mRS) at 6 months (mRS Score 0-3 favorable vs 4-6 unfavorable).

Results. Overall, $61(77.2 \%)$ of 79 patients who did and $292(34 \%)$ of the 860 patients who did not undergo DC had a poor clinical grade on admission (World Federation of Neurosurgical Societies Grade IV-V, p < 0.0001). A favorable outcome was attained in $21(26.6 \%)$ of 79 patients who had undergone DC. In a comparison of favorable outcomes in patients with primary $(28.0 \%)$ or secondary DC $(25.5 \%)$, no difference could be found $(\mathrm{p}=0.8)$. Subgroup analysis with respect to the underlying indication for DC (brain swelling vs bleeding vs infarction) revealed no difference in the rate of favorable outcomes. On multivariate analysis, acute hydrocephalus $(\mathrm{p}=0.009)$ and clinical signs of herniation $(\mathrm{p}=0.02)$ were significantly associated with an unfavorable outcome.

Conclusions. Based on the data in this study the authors concluded that primary as well as secondary craniectomy might be warranted, regardless of the underlying etiology (hemorrhage, infarction, or brain swelling) and admission clinical grade of the patient. The time from the onset of intractable intracranial pressure to DC seems to be crucial for a favorable outcome, even when a DC is performed late in the disease course after SAH. (DOI: 10.3171/2009.3.FOCUSO954)
\end{abstract}

\section{KeY WoRds - decompressive craniectomy - intracranial aneurysm • subarachnoid hemorrhage • intracerebral hemorrhage • brain swelling}

$\mathrm{D}$ ECOMPRESSIVE craniectomy lowers elevated ICP in patients with an intractable increase in pressure following brain trauma or cerebral infarction and improves outcome in patients with large territorial infarctions of the MCA.4,14,16

In patients with aneurysmal SAH, brain swelling can occur very early after the ictus ("primary") and later in the course of the disease as the result of complications associated with SAH ("secondary"; for example, cerebral infarction or bleeding). Regardless of its origin, brain swelling is known to worsen outcomes following $\mathrm{SAH} ., 7$ Its medical treatment is highly significant and often effective, although it can also be associated with severe side effects. Because of the unknown functional recovery, especially in poor-clinical-grade patients with SAH and

\footnotetext{
Abbreviations used in this paper: $\mathrm{ACoA}=$ anterior communicating artery; $\mathrm{DC}=$ decompressive craniectomy; $\mathrm{DHC}=$ decompressive hemicraniectomy; DW = diffusion weighted; ICA = internal carotid artery; ICH = intracerebral hematoma; ICP = intracranial pressure; $\mathrm{MCA}=$ middle cerebral artery; $\mathrm{mRS}=$ modified Rankin Scale; $\mathrm{PW}=$ perfusion weighted; $\mathrm{SAH}=$ subarachnoid hemorrhage; WFNS $=$ World Federation of Neurosurgical Societies .
}

associated elevated ICP, the indication for aggressive surgical treatment options such as DC is controversial. ${ }^{5,6,13}$ Recent literature dealing with DC has included patients with ICH and SAH only ${ }^{6}$ or had excluded patients treated with endovascular coiling. ${ }^{5}$ There are sparse data concerning the etiology of the mass effect that leads to DC-such as a space-occupying infarct, an ICH, or brain swelling without bleeding or infarction-which might per se significantly affect outcome. ${ }^{8,13}$

The purpose of the present analysis was to update another recent study in providing comprehensive clinical material ${ }^{9}$ and to analyze outcomes in a larger cohort of consecutive patients with primary and secondary DC, stratified according to the different underlying pathologies - that is, bleeding, infarction, or brain swelling-to find predictors that may guide treatment.

Parts of the clinical material have been published in a predecessor study. ${ }^{9}$

\section{Methods}

We studied 939 consecutive patients with aneurysmal SAH confirmed on CT or lumbar puncture and angiography between June 1999 and June 2008. During this 


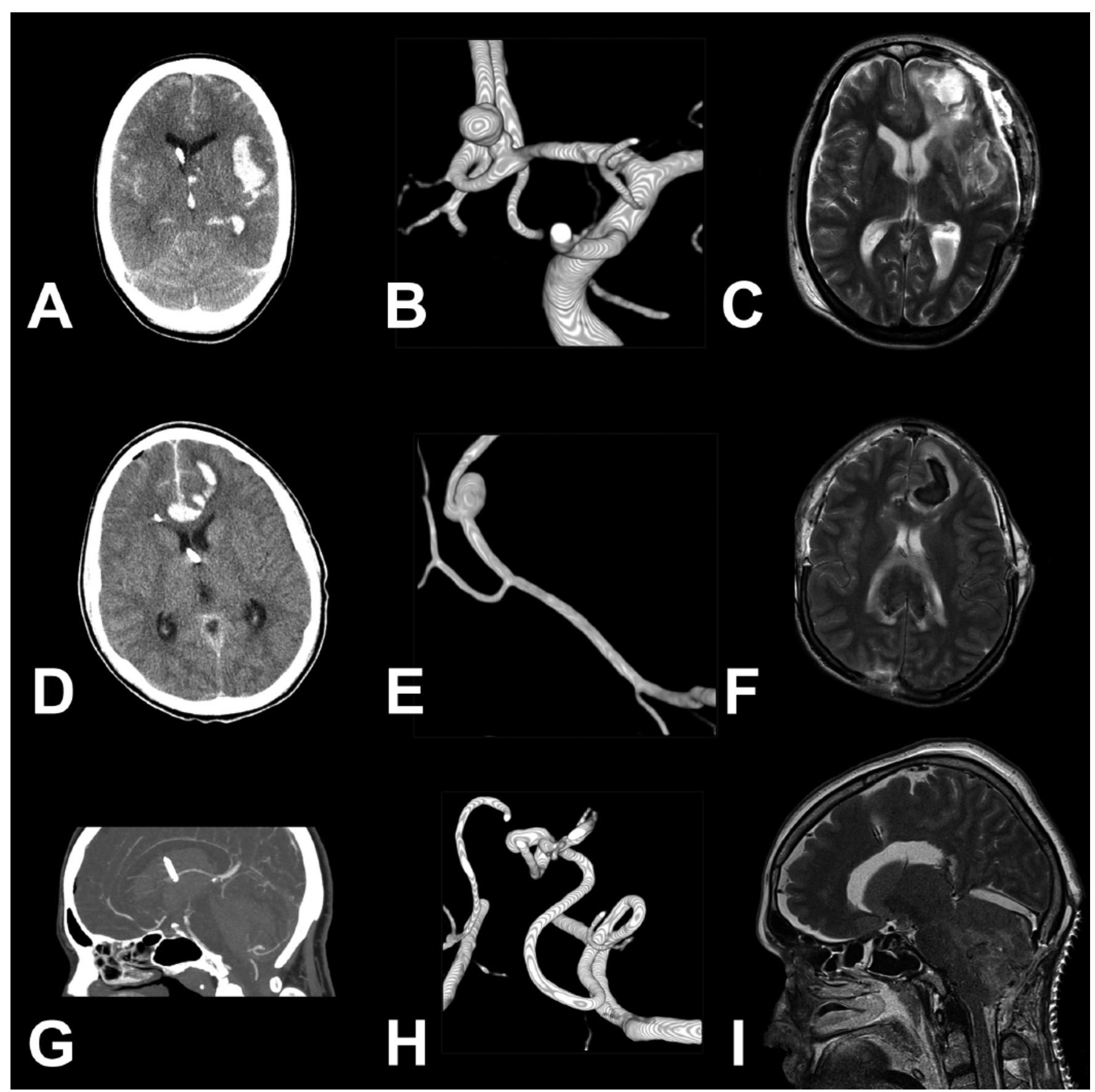

FIG. 1. Modalities of DC. Computed tomography scans showing SAH and ICH caused by ACoA (A), pericallosal artery (D), and PICA aneurysms $(G)$; the causative lesions can be seen on digital subtraction angiograms ( $B, E$, and $H$, respectively). Magnetic resonance images obtained after DHC (C), bifrontal craniectomy $(F)$, and suboccipital craniectomy (I) for intractably elevated ICP, demonstrating the extent of injury caused by SAH and the decompression achieved by DC.

period, 79 patients underwent DC as a treatment for elevated ICP. Information, including patient characteristics on admission and during the treatment course, treatment modality, size and location of the ruptured aneurysm, radiological features, presence and size of the ICH, presence of infarction, rebleeding, and the specific indication for DC, was prospectively entered into an SPSS database (SPSS, version 15, SAS Institute, Inc.). The treatment decision (coiling or clipping) was based on an interdisciplin- ary approach in each case. We followed an early surgery strategy whenever possible (within 24 hours of the onset of SAH) in patients of all clinical grades except in those who were hemodynamically unstable or moribund. Acute hydrocephalus was treated with external CSF diversion. Osmotherapy and mild hyperventilation were used for the medical treatment of elevated ICP. Our emphasis was to establish high normal euvolemia, and in cases of symptomatic cerebral vasospasm hemodynamic therapy was 
Decompressive craniectomy in subarachnoid hemorrhage

TABLE 1: Subgroups of 79 patients treated with DC

\begin{tabular}{|c|c|c|c|c|}
\hline \multicolumn{5}{|c|}{ DC Groups } \\
\hline \multicolumn{2}{|c|}{ Primary } & \multicolumn{3}{|c|}{ Secondary } \\
\hline 1 & 2 & 3 & 4 & 5 \\
\hline brain swelling w/o ICH & brain swelling w/ ICH & $\begin{array}{l}\text { brain swelling } \\
\text { w/o infarction or } \\
\text { rebleeding (SAH) }\end{array}$ & $\begin{array}{l}\text { brain swelling w/ signs of } \\
\text { infarction }\end{array}$ & $\begin{array}{l}\text { brain swelling w/ signs } \\
\text { of rebleeding(SAH) }\end{array}$ \\
\hline
\end{tabular}

instituted. ${ }^{12}$ Patients with impeding cerebral ischemia and proximal vasospasm were treated with balloon angioplasty $^{2}$ guided by PW and DW MR imaging.

\section{Surgical Technique}

In patients with persistent and medically refractory ICP values $>20 \mathrm{~mm} \mathrm{Hg}$, DC was performed by removing a large bone flap at the involved site. During DHC, a large frontotemporoparietooccipital flap extending from the supraorbital rim to just behind the lambdoid suture and from the temporal base to close $(1.5 \mathrm{~cm})$ to the midline was removed. The temporal bone was removed osteoclastically down to the base of the middle cerebral fossa. The size of the craniectomy was at least $15 \mathrm{~cm}$ in diameter. During bifrontal craniectomy, a large bone flap extending from the roots of the zygoma bilaterally and from $\sim 1 \mathrm{~cm}$ parallel and superior to the orbital rim to just about the coronal suture was removed in 1 piece. Craniectomy of the posterior cranial fossa was performed via a midline approach, with the removal of a bone flap extending from the lower margin of the transverse sinus to the foramen magnum. The lateral extensions were close $(\sim 1 \mathrm{~cm})$ to the expected margins of the sigmoid sinus. During each craniectomy the dura mater was opened widely, and the opening was extended to the sinus and bone margins in a stellate fashion (Fig. 1).

Patients were stratified into 5 groups (Table 1). In the primary DC groups the bone flap was not reinserted but instead was enlarged after surgical aneurysm obliteration in patients with signs of massive brain swelling without (Group 1) or with (Group 2) an ICH. Additionally, external ventricular drains or ICP (Spiegelberg) or brain tissue $\mathrm{PO}_{2}$ probes (Raumedic) were inserted in selected patients. Persons with elevated ICP $(>20 \mathrm{~mm} \mathrm{Hg}$ ) despite medical therapy underwent secondary DC. All of those undergoing secondary DC had already been treated for the ruptured aneurysm. Indications for secondary DC were intractable elevated ICP $(>20 \mathrm{~mm} \mathrm{Hg})$ without radiological signs of rebleeding (SAH) or infarction (Group 3) and elevated ICP with signs of infarction (Group 4) or rebleeding (repeat SAH in patients with coagulopathy, Group 5).

In all patients in Group 5, rebleeding consisted of $\mathrm{SAH}$, not ICH. Rebleeding was confirmed on $\mathrm{CT}$ as a larger SAH volume than before. All of these patients had coagulopathy-for example, warfarin before aneurysm rupture due to cardiac arrhythmia, artificial cardiac valve, or deep vein thrombosis.

Apart from close neurological monitoring, routine surveillance included daily transcranial Doppler mea- surements of red blood cell flow velocities and, in selected cases, multimodal monitoring of parenchymal brain tissue $\mathrm{PO}_{2}$, regional cerebral blood flow (thermodilution microprobe), interstitial metabolites (microdialysis), and PW/ DW MR imaging. Computed tomography was routinely performed 1) 24-48 hours after aneurysm clip application or coil obliteration to assess procedural complications, 2) on Day 14-21 to diagnose vasospastic infarctions and assess the necessity of a ventriculoperitoneal shunt, and 3) at various time points whenever neurological deteriorations occurred. Magnetic resonance imaging, including PW/DW imaging, ${ }^{2}$ was performed on Day 7, and cerebral angiography between Days 7 and 10 .

Sufficient fluid was administered to maintain a high normal euvolemic status. All patients received nimodipine from the day of admission. In the case of hyponatremia, fludrocortisone was added to the therapy. Desmopressin was used to control excessive diuresis. In cases of symptomatic vasospasm hypervolemia was instituted, and hypertension was induced with catecholamines. ${ }^{12}$ When hypertensive-hypervolemic-hemodilution therapy failed to improve delayed ischemic neurological deficit symptoms, patients with focal vasospasm were selected to undergo angioplasty. ${ }^{2}$ Outcome was assessed according to the mRS 6 months after treatment. Patients were divided into good-grade (WFNS Grade I-III) versus poor-grade (WFNS Grade IV-V) groups on admission and stratified into favorable (mRS Score 0-3) versus unfavorable (mRS Score 4-6) outcomes.

Statistical analysis was performed for all patients who underwent DC. An unpaired t-test was used for parametric statistics. Categorical variables were analyzed in contingency tables using the Fisher exact test. Results with a $p<0.05$ were considered statistically significant. In a second step, multivariate analysis was performed to find independent predictors of outcome at 6 months after discharge by using a binary logistic regression analysis and to find the confounding effects between potentially independent predictors. Variables with significant probability values on univariate analysis were considered potentially independent variables on multivariate analysis. A backward stepwise method was used to construct multivariate logistic regression models with the inclusion criterion of $\mathrm{p}<0.05$. All calculations were made with standard commercial software (SPSS Institute, Inc.).

\section{Results}

\section{Patient Characteristics}

Patient characteristics, including age, sex, clinical 
E. Güresir et al.

TABLE 2: Summary of patient characteristics on admission*

\begin{tabular}{|c|c|c|c|c|}
\hline \multirow[b]{2}{*}{ Characteristic } & \multicolumn{4}{|c|}{ №. $(\%)$} \\
\hline & w/o DC & $w / D C$ & $\mathrm{OR}(95 \% \mathrm{Cl})$ & $p$ Valuet \\
\hline no. of patients & 860 & 79 & & \\
\hline mean age in yrs & $54.0 \pm 14.2$ & $51.0 \pm 12.3$ & & 0.07 \\
\hline mean WFNS grade & $\|$ & V & & $<0.0001$ \\
\hline cases w/ poor WFNS grade (IV-V) & $292(34)$ & $61(77.2)$ & $6.6(3.8-11.4)$ & $<0.0001$ \\
\hline female sex & $510(59)$ & $59(74.7)$ & $2.0(1.2-3.4)$ & 0.007 \\
\hline cases $\mathrm{w} / \mathrm{ICH}<50 \mathrm{~cm}^{3}$ & $84(9.8)$ & $15(19)$ & $2.1(1.2-4.0)$ & 0.02 \\
\hline cases $\mathrm{w} / \mathrm{ICH}>50 \mathrm{~cm}^{3}$ & $46(5.3)$ & $22(27.8)$ & $6.8(3.8-12.1)$ & $<0.0001$ \\
\hline Fisher grade & 3 & 3 & & 0.9 \\
\hline mean time from ictus to aneurysm obliteration in hrs & $83.3 \pm 11$ & $51.2 \pm 18$ & & $<0.0001$ \\
\hline cases w/ rebleeding before treatment & $66(7.7)$ & $14(17.7)$ & $2.6(1.4-4.9)$ & 0.005 \\
\hline cases w/ acute hydrocephalus & $474(55.1)$ & $43(54.4)$ & & 0.9 \\
\hline cases of clipping & $278(32.3)$ & $53(67.1)$ & $4.2(2.6-7.0)$ & $<0.0001$ \\
\hline mean aneurysm size in $\mathrm{mm}$ & $6 \pm 4.2$ & $11 \pm 7$ & $5(3.9-6.0)$ & $<0.0001$ \\
\hline cases of shunt placement after 6 mos & $121(14.1)$ & $20(25.3)$ & $2.1(1.2-3.6)$ & 0.01 \\
\hline mean mRS score & 2 & 5 & & $<0.0001$ \\
\hline cases w/ unfavorable outcome (mRS 4-6) & $238(27.7)$ & $59(74.7)$ & $7.7(4.5-13.1)$ & $<0.0001$ \\
\hline
\end{tabular}

* Values are presented as the means \pm SDs, unless indicated otherwise.

+ Fisher exact test.

status on admission, and angiographic, and CT imaging findings are shown in Table 2. Of 939 patients, 353 $(37.6 \%)$ presented with a poor clinical grade. Sixty-one of the poor-grade patients (17.3\%) underwent DC. Among patients admitted in a good clinical grade, DC was performed in 18 (3.1\%) of 586 patients $(\mathrm{p}<0.0001, \mathrm{OR}=6.6$, 95\% CI 3.8-11.4).

Age did not differ between patients with $(51 \pm 12.3$ years) or without $(54.0 \pm 14.2$ years) $\mathrm{DC}(\mathrm{p}=0.07)$. The rate of women was higher in the group treated with DC compared with the group not treated with DC (74.7 vs $59 \%, \mathrm{p}=0.007, \mathrm{OR}=2.0,95 \% \mathrm{CI} 1.2-3.4)$. Microsurgical clipping was performed in $67.1 \%$ of patients treated with DC compared with $32.3 \%$ patients not treated with DC $(\mathrm{p}<0.0001, \mathrm{OR}=4.2,95 \%$ CI 2.6-7.0). Four hundred seventy-four $(55.1 \%)$ of 860 patients who did not undergo DC and 43 (54.4\%) of 79 patients who did undergo DC presented with acute hydrocephalus $(\mathrm{p}=0.9)$.

\section{Aneurysm Size and Site}

In patients treated with DC the most frequent site for a ruptured aneurysm was the MCA (55.0\%) and the ICA (18.3\%). Anterior communicating artery aneurysms caused SAH in $15.0 \%$ of patients who underwent DC (Table 3). In the group of patients that did not undergo DC, aneurysms were more frequently located at the ACoA $(33.4 \%, \mathrm{p}=0.003, \mathrm{OR}=2.8,95 \%$ CI $1.3-5.9)$ and less frequently at the MCA $(17.0 \%, \mathrm{p}<0.0001, \mathrm{OR}=5.9,95 \%$ CI 3.4-10.3). There was no difference between groups the DC groups in terms of bleeding at the ICA (23.2 vs $18.3 \%)$. Patients treated with DC had significantly larger aneurysms (mean: 11 vs $6 \mathrm{~mm}, \mathrm{p}<0.0001, \mathrm{OR}=5,95 \%$ CI 3.9-6.0; Table 2).

\section{Decompressive Craniectomy Group}

Of the 79 patients treated with DC, $61(77.2 \%)$ presented in a poor grade. Decompressive hemicraniectomy was performed as a primary procedure in 32 patients $(40.5 \%)$ and as a secondary procedure in $47(59.5 \%)$. According to the indication for DC, in only 3 patients (3.8\%) with clear signs of brain swelling during aneurysm obliteration, the bone flap was not reinserted, but the craniotomy site was enlarged instead (Group 1: primary DC without ICH). Twenty-nine patients (36.7\%) underwent primary DC because of brain swelling together with an ICH at presentation (Group 2). Sixteen patients (20.3\%) were treated with secondary DC due to intractable ICP without infarcts (Group 3). In 23 patients (29.1\%) secondary DC was performed due to space-occupying infarcts (Group 4), and 8 patients (10.1\%) underwent secondary DC after rebleeding caused by coagulopathy (SAH, Group 5).

\section{Decompressive Craniectomy Modality}

Depending on the site of the space-occupying lesion, a right hemispheric DHC was performed in 36 cases and a left hemispheric DHC in another 36 cases. A bifrontal craniectomy was performed in 6 cases and a DC of the posterior fossa in 1 case. In comparing the rate of favorable outcomes in patients undergoing DHC (25.0\%) and bifrontal DC (16.7\%), no difference could be found ( $\mathrm{p}=$ 1.0).

\section{Appropriate Time for DC}

In all patients who underwent a primary DC, decompression was performed on the day of the SAH. The mean time to surgery was $4.5 \pm 5.7$ hours. In patients who underwent secondary DC, decompression was performed 
Decompressive craniectomy in subarachnoid hemorrhage

TABLE 3: Aneurysm site in 79 cases*

\begin{tabular}{ccccc}
\hline & w/0 DC & w/ DC & & \\
Aneurysm Site & (\% cases) & (\% cases) & OR $(95 \%$ Cl $)$ & p Value $\dagger$ \\
\hline ant circulation & & & & \\
ACoA & 33.4 & 15.0 & $2.8(1.3-5.9)$ & 0.003 \\
ACA & 4.4 & 6.7 & & 0.3 \\
ICA & 23.2 & 18.3 & & 0.4 \\
MCA & 17.0 & 55.0 & $5.9(3.4-10.3)$ & $<0.0001$ \\
pst circulation & 22.0 & 5.0 & & 0.2 \\
\hline
\end{tabular}

* $\mathrm{ACA}=$ anterior cerebral artery.

$\uparrow$ Fisher exact test.

$3.3 \pm 3.1$ days $(79.2 \pm 73$ hours $)$ after SAH. The mean time to aneurysm obliteration was $26 \pm 25.8$ hours after ictus in patients who underwent secondary DC. Hence, the time to aneurysm treatment was significantly shorter in the group of patients that underwent primary DC compared with the group that underwent secondary DC $(\mathrm{p}<$ 0.001).

\section{Treatment Outcome}

Treatment outcome was stratified according to the 5 DC groups (Table 4). Overall, 21 (26.6\%) of 79 patients achieved a favorable outcome. No patients in Group 1 and $9(31.0 \%)$ of 29 patients in Group 2 attained favorable outcomes. Six (37.5\%) of 16 patients in Group 3, 5 (21.7\%) of 23 in Group 4, and 1 (12.5\%) of 8 in Group 5 had favorable outcomes (Fig. 2). In comparing the rate of favorable outcomes in patients treated with primary $(28.0 \%)$ and secondary DC (25.5\%), no difference could be found $(\mathrm{p}=0.8)$.

There was no difference in favorable outcomes between patients in Groups 2 and 5 (brain swelling with $\mathrm{ICH}$ or repeat $\mathrm{SAH}$ ) and those in Group 4 (brain swelling with infarction; $p=0.8$ ). Furthermore, no difference in favorable outcomes could be found in a comparison of patients in Groups 1 and 3 (brain swelling without additional lesion on CT), and those in Groups 2, 4, and 5 (brain swelling with $\mathrm{ICH}$, repeat $\mathrm{SAH}$, or infarcts; $\mathrm{p}=$ $0.6)$.

The mortality rates 6 months after treatment were $40 \%$ in patients who had undergone primary DC and $21.7 \%$ in those who had undergone secondary DC $(\mathrm{p}=$ $0.3)$.

In poor-grade patients with a favorable outcome, the time to aneurysm obliteration was $3.9 \pm 1.8$ hours compared with $15.2 \pm 13.9$ hours in poor-grade patients with an unfavorable outcome $(p=0.01)$. Eight of the 15 poorgrade patients with a favorable outcome had an additional ICH and underwent primary DC (53.3\%, Group 2), 3 patients underwent secondary DC without signs of infarctions (20\%, Group 3), and 4 patients underwent DC due to infarction (26.7\%, Group 4).

Overall, 42 of the 79 patients had clinical signs of tentorial herniation (mydriasis). Eight (19\%) of the 42 patients with and $11(18.9 \%)$ of 37 patients $(\mathrm{p}=0.3)$ without signs of cerebral herniation attained a favorable outcome.

Cranioplasty was performed in surviving patients with DHC and bifrontal craniectomy. The mean time from ictus to cranioplasty was $90 \pm 47$ days.

\section{Multivariate Analysis}

Using a backward stepwise method in a binary logistic regression model, the multivariate relationships were analyzed in patients with SAH and DC for the variable outcome at 6 months after discharge. Of the variables that influenced outcome 6 months after discharge on univariate analysis in patients with SAH who had undergone DC, acute hydrocephalus ( $\mathrm{p}=0.009$, OR 5.8, 95\% CI 1.5-21.9) remained, and clinical signs of cerebral herniation $(\mathrm{p}=$

TABLE 4: Summary of patient characteristics according to DC group

\begin{tabular}{|c|c|c|c|c|c|}
\hline \multirow[b]{2}{*}{ Parameter } & \multicolumn{5}{|c|}{ DC Group (No. [\%]) } \\
\hline & 1 & 2 & 3 & 4 & 5 \\
\hline no. of patients & 3 & 29 & 16 & 23 & 8 \\
\hline mean age in yrs & $43 \pm 6$ & $52.0 \pm 14$ & $48.5 \pm 9$ & $51.0 \pm 11.5$ & $52.0 \pm 11.6$ \\
\hline mean WFNS grade & 4 & 5 & 4 & 4 & 5 \\
\hline cases w/ poor WFNS grade (IV-V) & 1 & $28(96.5)$ & $10(62.5)$ & $16(69.6)$ & 6 \\
\hline female sex & 2 & $22(75.9)$ & $13(81.3)$ & $18(78.3)$ & 6 \\
\hline cases w/ acute hydrocephalus & 1 & $8(27.6)$ & $11(68.8)$ & $16(69.6)$ & 7 \\
\hline cases w/ clipping & 2 & $22(75.9)$ & $7(43.8)$ & $16(69.6)$ & 6 \\
\hline cases w/ angiographic vasospasm ( $\geq 60 \%)$ & 0 & 8 & 5 & 23 & 8 \\
\hline cases w/ shunt placement at 6 mos after treatment & 2 & $3(10.3)$ & $8(50)$ & $7(30.4)$ & 0 \\
\hline mean mRS score at 6 mos after treatment & 6 & 5 & 4 & 5 & 6 \\
\hline cases w/ unfavorable outcome (mRS 4-6) & 3 & $20(69)$ & $10(62.5)$ & $18(78.3)$ & 7 \\
\hline cases w/ unilat dilated pupil & 1 & $12(41.4)$ & $3(18.8)$ & $15(65.2)$ & 1 \\
\hline cases w/ bilat dilated pupils & 0 & $4(13.8)$ & $2(12.5)$ & $2(8.7)$ & 2 \\
\hline cases w/o dilated pupils & 2 & $13(44.8)$ & $11(68.7)$ & $6(26.1)$ & 5 \\
\hline
\end{tabular}




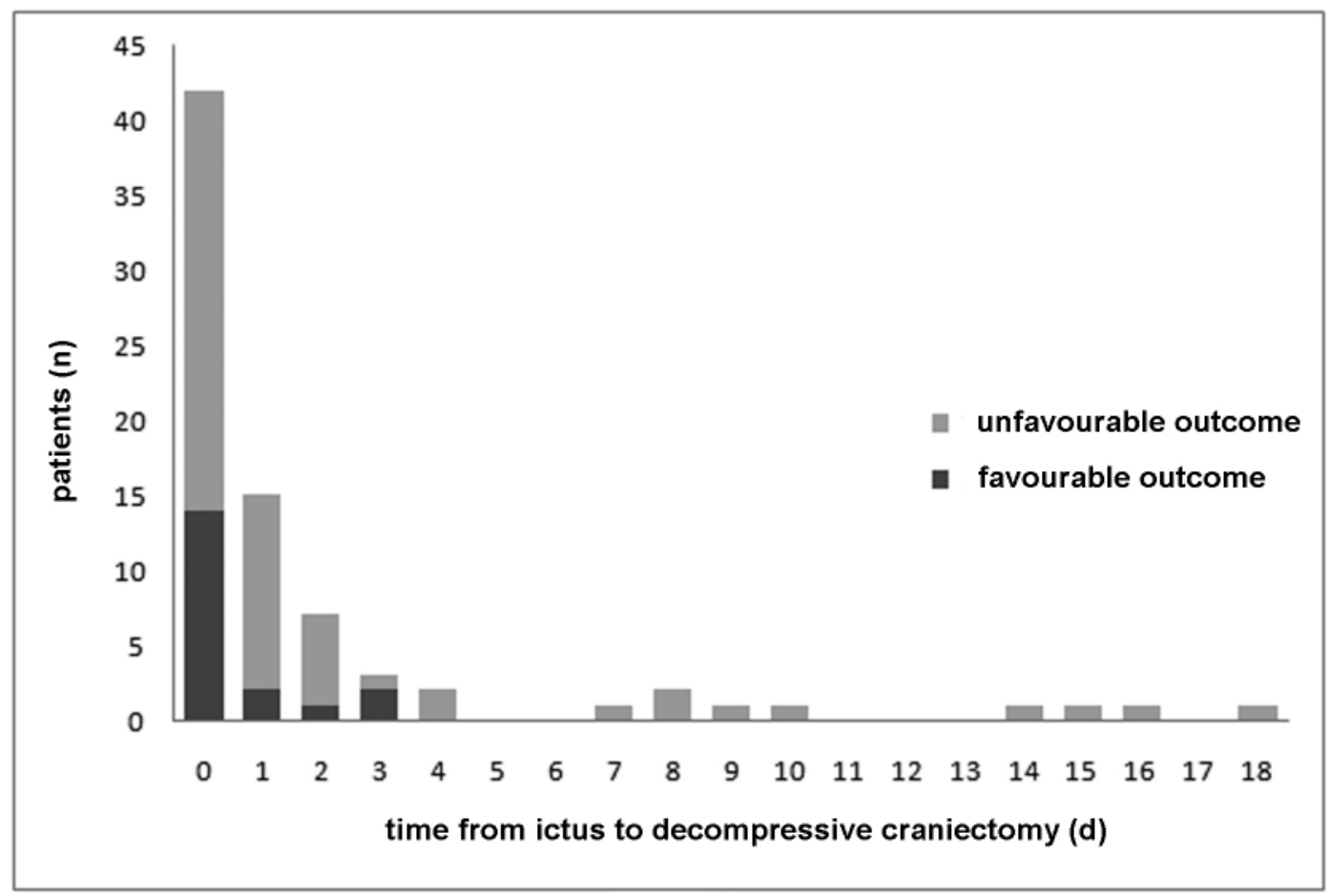

FIG. 2. Bar graph showing the timing and outcome of DHC. $d=$ day; $n=$ number.

0.02, OR 3.1, 95\% CI 1.1-8.1) became significant in the multivariate regression model (Nagelkerke $R^{2}=0.33$ ).

\section{Discussion}

The role of DC in patients with refractory elevated ICP following traumatic brain injury or cerebral infarction is beneficial. ${ }^{1,411,14,17}$ Decompressive craniectomy leads to a 2-step reduction in elevated ICP after bone flap removal and dural opening as well as improvement in tissue perfusion and oxygenation. ${ }^{10}$ However, authors of only a few studies have dealt with DC in patients with aneurysmal SAH. To the best of our knowledge, we have presented the largest series of patients with aneurysmal SAH who underwent DC. To improve treatment decision making, we placed special emphasis on the clinical settings that indicated primary or secondary DC; therefore, we further stratified our patients according to their underlying pathology, for example, bleeding, infarction, or brain swelling.

In this study of 939 consecutive patients the data accumulation was prospective, whereas the analysis was retrospective. Outcome in the 79 patients who had undergone DC was favorable in $21(26.6 \%)$, despite the presence of space-occupying lesions (ICH or infarct), signs of clinical herniation (mydriasis in $53.2 \%$ of the cases), and a population of $77.2 \%$ with poor-grade SAH (WFNS $\mathrm{IV}-\mathrm{V}$ ) on admission. There was no difference in the rate of favorable outcomes between patients who underwent primary $(29 \%)$ and secondary DC $(26.1 \%$; p $=0.8)$. Our results indicated that DC may be warranted regardless of whether the patient suffers from bleeding, infarction, or brain swelling.
According to the data of Schirmer et al., ${ }^{13} 5$ (31\%) of 16 patients were treated with primary and $11(69 \%)$ with secondary DC. A favorable outcome was attained in 4 $(80 \%)$ of the 5 patients who had undergone primary and $3(27 \%)$ of the 11 patients who had undergone secondary DC. Four of the 5 patients with primary DC had an additional $\mathrm{ICH}$ on admission. The patients with $\mathrm{ICH}$ in that study had a smaller hematoma volume $\left(<50 \mathrm{~cm}^{3}\right)$ in contrast to a rate of $27.8 \%$ of patients with hematomas larger than $50 \mathrm{~cm}^{3}$ in our current series, which may have contributed to a better outcome. In addition, Schirmer et al. reported no patients with a Fisher Grade $3 \mathrm{SAH}$ on admission, which translated into a lower risk of vasospasm and consecutive infarctions. Indeed, these authors described no patients who underwent DC for space-occupying infarcts. Our population consisted of $69.6 \%$ patients with Fisher Grade 3 SAHs, and $29.1 \%$ underwent DC because of infarcts; nevertheless, $21.7 \%$ of them had a favorable outcome. In other words, we showed that a relevant number of even those patients with high-grade SAHs as well as additional large, space-occupying ICHs and those with Fisher Grade 3 bleeding and infarcts can have favorable outcomes.

Buschmann et al. ${ }^{5}$ have treated 38 patients with $\mathrm{SAH}$ by using DC for intractable ICP and have found $52.6 \%$ favorable outcomes after 12 months. According to their published data, $76.2 \%$ of the patients treated with primary DC had Fisher Grade 4 SAHs, but no information about ICH size was given. Sixty percent of the patients who had undergone secondary DC for a postoperative epidural or subdural hematoma attained a favorable outcome. This result might explain the good outcome in their study group given the extraaxial nature of the lesion and the possibil- 


\section{Decompressive craniectomy in subarachnoid hemorrhage}

ity of a quick ICP reduction following hematoma evacuation. No DC was performed as a result of epi- or subdural hematomas in the current study. Moreover, $84.3 \%$ of patients had a poor clinical grade on admission, and $83.3 \%$ of those without and $16.7 \%$ of those with infarctions attained a favorable outcome. Among the subgroup of patients without infarctions, $80 \%$ had Fisher Grade 4 SAHs. Again, no data on ICHs or intraventricular hematomas size were given, which would be important for a comparison of outcomes.

In our series the subgroup that underwent secondary DC for space-occupying infarctions attained a comparable or slightly better rate of $21.7 \%$ favorable outcomes. Careful decision making is needed in this group of patients with per se poor prognoses due to large infarctions. D'Ambrosio et al. ${ }^{6}$ have analyzed 12 patients with ICHs on admission and found that $33.3 \%$ had favorable outcomes, a rate somewhat higher than our $31.0 \%$. Note, however, that D'Ambrosio and colleagues' series included only $8.3 \%$ large ICHs $\left(>50 \mathrm{~cm}^{3}\right)$ versus $27.8 \%$ in our study population.

Altogether, it is not surprising that the outcome in severely ill patients undergoing DC in smaller series varies, especially because of different patient characteristics on admission (for example, WFNS grade, Fisher grade, and accompanying $\mathrm{ICH}$ ). It is intriguing that outcome in the current study is comparable among the DC groups, regardless of the different underlying pathologies leading to DC (ICH, brain swelling, or infarction). An overall favorable outcome of $25.3 \%$ in the subset of critically ill patients with life-threatening conditions, in which a general conservative approach is usually accepted because of an expected poor prognosis, in our opinion is encouraging and warrants aggressive therapy in the future.

We believe that DC is useful in lowering intractably elevated ICP regardless of the aneurysm location (Fig. 1). The predominance of patients with MCA aneurysms undergoing DC in our series might have been attributable to the higher rate of large ICHs caused by MCA aneurysms compared with aneurysms of other locations as previously described. ${ }^{8}$

On multivariate analysis, we found that early hydrocephalus $(p=0.009)$ and clinical signs of herniation-for example, mydriasis $(\mathrm{p}=0.02)$-were associated with an unfavorable outcome. The association between cerebral herniation and poor outcome is not surprising and has been addressed by other authors. ${ }^{5,13}$ Smith et al. ${ }^{15}$ have even proposed the use of a prophylactic DC for the treatment of patients with poor-grade $\mathrm{SAH}$ and an additional $\mathrm{ICH}$. Although the optimal time point for DC must still be defined, an early craniectomy seems to be beneficial, leading to favorable outcomes in $25.3 \%$ of cases. This result is corroborated by the significantly poorer outcome in a heterogeneous group of patients with signs of cerebral herniation due to different underlying pathologies (brain swelling, infarct, and ICH). The result of univariate analyses showing no effect of mydriasis on outcome was somewhat surprising. However, the multivariate analysis, with its better control for confounding factors, showed that the presence of mydriasis indeed was 1 factor that significantly influenced outcome. The correlation between acute hydrocephalus and poor outcome has been addressed elsewhere. ${ }^{18}$ Mortality rates as well as cerebral infarction rates are higher in patients with acute hydrocephalus, as compared with the rates in patients without, as described by van Gijn et al. ${ }^{18}$ Therefore, it is not surprising that on multivariate analyses in the current series, acute hydrocephalus was 1 of the factors determining outcome.

\section{Study Limitations}

The present study has several limitations. The data analysis was performed retrospectively. Patients were not randomized for the treatment or control groups. Even though the patient series in the current study represents the largest thus far to suffer from aneurysmal SAH and to be treated with DC, our statistical analysis was still handicapped.

Altogether, we provide data showing 1) that the underlying pathology does not seem to limit outcome after $\mathrm{DC}$, and 2) that the time from the initial SAH to DC is not relevant. Instead, the time from the onset of elevated ICP - whether due to bleeding, infarction, or brain swelling-seems to be crucial for patient outcome. This conclusion is corroborated by the finding that cerebral herniation significantly affects outcome.

\section{Conclusions}

According to our data, DC is a valid option in the treatment of patients with aneurysmal SAH and intractable ICP. Decompressive craniectomy can be indicated early or late in the course after SAH if performed immediately after the onset of intractable ICP.

A favorable outcome can be attained in one-quarter of these patients. Even in the subgroup of patients with infarcts or large hematomas for which the prognosis seems limited, DC might be warranted. Nevertheless, careful decision making is needed for each patient, especially when signs of cerebral herniation have persisted for a long time. It is important for clinical decision making that DC may be indicated regardless of the underlying pathophysiology_bleeding, infarction, or brain swelling - and the admission grade of the patients.

\section{Disclosure}

The authors report no conflict of interest concerning the materials or methods used in this study or findings specified in this paper.

\section{References}

1. Albanese J, Leone M, Alliez JR, Kaya JM, Antonini F, Alliez B, et al: Decompressive craniectomy for severe traumatic brain injury: evaluation of the effects at one year. Crit Care Med 31:2535-2538, 2003

2. Beck J, Raabe A, Lanfermann H, Berkefeld J, De Rochemont Rdu M, Zanella F, et al: Effects of balloon angioplasty on perfusion- and diffusion-weighted magnetic resonance imaging results and outcome in patients with cerebral vasospasm. J Neurosurg 105:220-227, 2006

3. Broderick JP, Brott TG, Duldner JE, Tomsick T, Leach A, et al: Initial and recurrent bleeding are the major causes of death 


\section{E. Güresir et al.}

following subarachnoid hemorrhage. Stroke 25:1342-1347, 1994

4. Bullock MR, Chesnut R, Ghajar J, Gordon D, Hartl R, Newell DW, et al: Surgical management of acute subdural hematomas. Neurosurgery 58 (3 Suppl):S16-S24, 2006

5. Buschmann U, Yonekawa Y, Fortunati M, Cesnulis E, Keller E, et al: Decompressive hemicraniectomy in patients with subarachnoid hemorrhage and intractable intracranial hypertension. Acta Neurochir (Wien) 149:59-65, 2007

6. D'Ambrosio AL, Sughrue ME, Yorgason JG, Mocco JD, Kreiter KT, Mayer SA, et al: Decompressive hemicraniectomy for poor-grade aneurysmal subarachnoid hemorrhage patients with associated intracerebral hemorrhage: clinical outcome and quality of life assessment. Neurosurgery 56:12-19, 2005

7. Fergusen S, Macdonald RL: Predictors of cerebral infarction in patients with aneurysmal subarachnoid hemorrhage. Neurosurgery 60:658-667, 2007

8. Güresir E, Beck J, Vatter H, Setzer M, Gerlach R, Seifert V, et al: Subarachnoid hemorrhage and intracerebral hematoma: incidence, prognostic factors, and outcome. Neurosurgery 63:1088-1094, 2008

9. Güresir E, Raabe A, Setzer M, Vatter H, Gerlach R, Seifert V, et al: Decompressive hemicraniectomy in subarachnoid hemorrhage: the influence of infarction, hemorrhage, and brain swelling. J Neurol Neurosurg Psychiatry [in press], 2009

10. Jaeger M, Soehle M, Meixensberger J: Effects of decompressive craniectomy on brain tissue oxygen in patients with intracranial hypertension. J Neurol Neurosurg Psychiatry 74:513-515, 2003

11. Juttler E, Schwab S, Schmiedek P, Unterberg A, Hennerici M, Woitzik J, et al: Decompressive Surgery for the Treatment of Malignant Infarction of the Middle Cerebral Artery (DESTINY): a randomized, controlled trial. Stroke 38:2518-2525, 2007

12. Raabe A, Beck J, Keller M, Vatter H, Zimmermann M, Seifert
V, et al: Relative importance of hypertension compared with hypervolemia for increasing cerebral oxygenation in patients with cerebral vasospasm after subarachnoid hemorrhage. J Neurosurg 103:974-981, 2005

13. Schirmer CM, Hoit DA, Malek AM: Decompressive hemicraniectomy for the treatment of intractable intracranial hypertension after aneurysmal subarachnoid hemorrhage. Stroke 38:987-992, 2007

14. Schwab S, Steiner T, Aschoff A, Schwarz S, Steiner HH, Jansen O, et al: Early hemicraniectomy in patients with complete middle cerebral artery infarction. Stroke 29:1888-1893, 1998

15. Smith ER, Carter BS, Ogilvy CS: Proposed use of prophylactic decompressive craniectomy in poor-grade aneurysmal subarachnoid hemorrhage patients presenting with associated large sylvian hematomas. Neurosurgery 51:117-124, 2002

16. Vahedi K, Hofmeijer J, Juettler E, Vicaut E, George B, Algra A et al: Early decompressive surgery in malignant infarction of the middle cerebral artery: a pooled analysis of three randomised controlled trials. Lancet Neurol 6:215-222, 2007

17. Vahedi K, Vicaut E, Mateo J, Kurtz A, Orabi M, Guichard JP, et al: Sequential-design, multicenter, randomized, controlled trial of early decompressive craniectomy in malignant middle cerebral artery infarction (DECIMAL Trial). Stroke 38:2506-2517, 2007

18. van Gijn J, Hijdra A, Wijdicks EF, Vermeulen M, van Crevel $\mathrm{H}$ : Acute hydrocephalus after aneurysmal subarachnoid hemorrhage. J Neurosurg 63:355-362, 1985

Manuscript submitted February 14, 2009.

Accepted March 20, 2009.

Address correspondence to: Erdem Güresir, M.D., Department of Neurosurgery, Johann Wolfgang Goethe-University, Frankfurt am Main, Schleusenweg 2-16, 60528 Frankfurt am Main, Germany. email: Gueresir@em.uni-frankfurt.de. 